\title{
Task-Specificity and Resource Allocation in Information Perception in Three-Dimensional Space
}

\author{
Antje Lichtenstein \\ Technische Universität Berlin, \\ Center of Human-Machine Systems, \\ Franklinstr. 28-29, 10587 Berlin, Germany \\ +49(0)30 314-29932 \\ Antje.Lichtenstein@mms.tu-berlin.de
}

\begin{abstract}
Modern technologies are more and more capable of presenting information in three-dimensional space instead of being limited to a standard two-dimensional desktop workstation. Thus, it becomes necessary to examine whether display location does have an effect on human information perception and processing. This paper presents two studies concerned with this subject. The first study examined whether optimal display location depends on the task, which is being executed. We found evidence for differences in tasks with different cognitive engagement levels. The second study aimed at a) examining task characteristics in more detail and b) answering the basic question whether it is generally beneficial to present concurrent information in dual-task situations in two instead of one perceptual depth plane in order to optimize resource utilization. Results support the notion of a two-plane benefit.
\end{abstract}

\section{Categories and Subject Descriptors}

H.1.2 [Models and Principles]: User/Machine Systems human factors, human information processing

\section{General Terms}

Performance, Design, Experimentation, Human Factors

\section{Keywords}

Human visual perception, information visualization, threedimensional space, 3D, dual-task, task-specificity, resource allocation, object detection, spatial cognition, augmented reality

\section{INTRODUCTION}

Human Computer Interaction (HCI) is more and more detaching from a standard 2D-desktop metaphor, utilizing more freely the space surrounding a user to present information or facilitate interaction. Thus, HCI research on alternative information presentation and interaction becomes necessary. An important question in this context is whether display location, especially location in depth, influences human information perception and processing, since traditional HCI research on information presentation has mainly dealt with

(c) The Author 2009.

Published by the British Computer Society location in 2D-space. Applied research on 3D visual perception so far focuses on virtual reality, presenting three-dimensional objects on 2D-desktops or in a cave. Three-dimensional vision is then achieved through auto-stereograms or shutter glasses, while data is actually presented in one depth plane only (e.g. [1],[2],[3]). In contrast, our project concentrates on human visual perception in real three-dimensional space. Project results shall be utilized to define design guidelines for HCI in $3 \mathrm{D}$ and may also be used to augment computational models on visual perception (e.g. [4], [5])

\subsection{Theoretical Background}

Integrating neuropsychological work on three-dimensional spatial interaction, Previc [4] suggested the division of the space surrounding an individual into four realms (see Figure 1). They consist of one peri-personal realm within hand-reaching distance and three extra-personal realms further away. Each realm has specific properties concerning neurological processing of stimuli and reactions, influencing human perception and behaviour. As far as visual perception is concerned, in the peri-personal realm global form, depth and motion are perceived, while extra-personal realms are utilized for visual search and object recognition (focal realm), navigation and target orientation (action realm) or postural control (ambient realm).

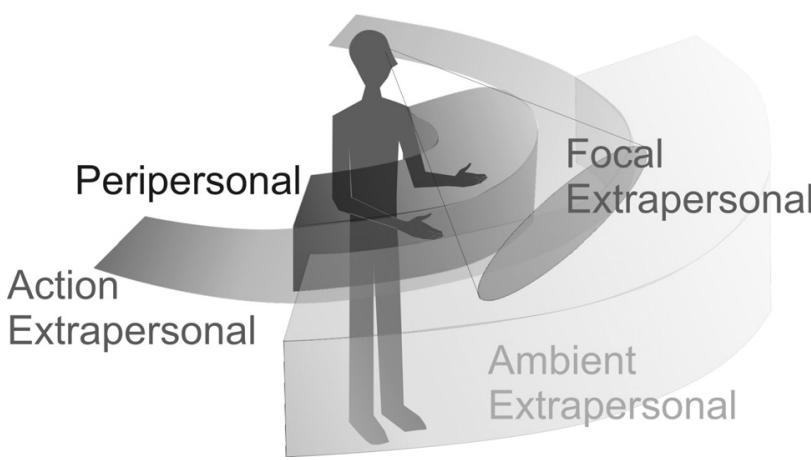

Figure 1. A theoretical model of 3D spatial interaction, adapted from Previc [2]

The model has been employed and supported mainly by neuropsychological research (e.g. [7], [8]). Studies in the field of cognitive psychology are rather scarce. However, it does appear to be highly useful in the field of HCI to form a foundation for research on augmented and virtual reality techniques deployed for presenting visual information in threedimensional space. 
Considering the differential neurological processing of information perceived in different perceptual realms, it is reasonable to assume that the optimal presentation location of certain information is dependent on the nature of the task that is to be performed. Following Norman [9], tasks can be defined as either experiential or reflective. Responses to tasks which are completed in experiential mode are automatic and not reflected upon, e.g. simple object detection. In contrast, reflective cognition utilizes conscious engagement in the task, e.g. planning or decision making. In the following, this definition will be used to test for task-specificity in 3D-visual information processing.

When two or more tasks are executed at the same time, equal or different in nature, 3D-technologies could be advantageous because of their ability to present concurrent visual information in different perceptual depths (i.e. realms). According to Wickens [10], perceptual resources are sensitive to presentation location. He describes that two focal tasks will interfere and lead to poorer performance while a focal and an ambient task can be completed in parallel due to improved resource allocation. This could be true for peripersonal and extrapersonal tasks also, which is another issue to be considered in the current research project and which is touched by the second experiment presented in this paper.

\section{EXPERIMENT 1}

The first experiment reported here was concerned with the issue of task-specific perception and processing of visual information in two different depth planes, which can be defined as peri- and extrapersonal perceptual realms, in general. Two different tasks had to be completed, one of experiential and one of reflective nature. The measure of interest was reaction time.

\subsection{Participants}

The test sample consisted of 45 subjects ( 30 female) with a mean age of 29.1 (age range: 20-65 years), which were recruited via PESA-subject database, a cooperation of Humboldt University - Department of Engineering Psychology and Berlin University of Technology - Center of Human Machine Systems.

\subsection{Procedure and Material}

Subjects were welcomed, briefed and then situated in front of the HoloPro ${ }^{\mathrm{TM}} 3$ i-PrOS Touch by pronova Projection Systems within hand-reaching distance. Two LCD projectors which were affixed to the ceiling were used to project visual information to either the transparent HoloPro or a wall approximately four meters apart from it (see Figure 2).

Before the dual-task trials started, subjects had to complete four baseline trials of two minutes-length each in order to get used to the setting and the tasks. The first baseline was a simple object detection task (experiential), where subjects had to react to white smiley pictures which appeared randomly at the transparent screen every one to nine seconds, by saying "yes" whenever they noticed one. Smileys then disappeared. The second baseline was the same task, but smileys were presented at the far screen.

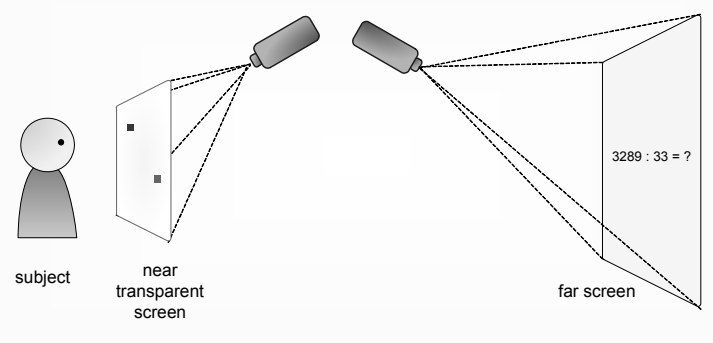

Figure 2. Experimental set-up

The third baseline was an arithmetic task (reflective), were subjects had to add random one-digit numbers. For this purpose they were given a mouse with two buttons and had to click one or the other button according to the correctness of a calculation result which was presented to them shortly after the second summand had disappeared. The numbers were presented on the far screen. The fourth baseline was the arithmetic task again, but numbers were presented on the near screen. After the baseline phase, two 3.5 min-long experimental trials followed where the object detection and the arithmetic tasks had to be completed in parallel. In trial one, smileys were presented on the near screen, while numbers were presented on the far screen. In trial two, screens were switched.

Both, baseline trials and experimental trials were randomized.

When subjects had completed the last trial, they were informed about the purpose of the experiment in more detail, rewarded and dismissed.

\subsection{Results}

Data was analysed using SPSS. Group means were compared via analysis of variance (ANOVA) and differences were further investigated using t-tests.

Means and standard deviations for reaction times in dual-task trials are reported in Table 1. As expected, there was a significant main effect of task $(F(1,44)=25.99, \mathrm{p}<.001$, partial $\left.\eta^{2}=.371\right)$, meaning that reaction times for the detection task were significantly smaller than those for the arithmetic task. There was no significant main effect of depth plane.

Table 1. Means and standard deviations (SD) for reaction times in seconds, experiment one

\begin{tabular}{|c|c|c|}
\hline & Mean & SD \\
\hline Detection task near & .908 & .173 \\
\hline Detection task far & .987 & .275 \\
\hline Arithmetic task near & 1.300 & .487 \\
\hline Arithmetic task far & 1.157 & .477 \\
\hline
\end{tabular}

The interaction between task and depth plane was significant $\left(F(1,44)=11.4, \mathrm{p}<.01\right.$, partial $\left.\eta^{2}=.206\right)$. T-tests revealed that for both, the detection task $(t(44)=-2.52, \mathrm{p}<.025)$ and the arithmetic task $(t(44)=-3.07, \mathrm{p}<.01)$, depth plane had an effect on reaction times: The detection task was solved faster in the near plane, while the arithmetic task was solved faster in the far plane. 
Note that this effect did not show up in the baseline trials one and two, where tasks were executed singularly. Thus, it only seems to appear when resources are scarce. Baselines three and four could not be compared due to a technological problem which led to the loss of baseline four data.

\subsection{Discussion}

The first experiment showed that human information perception and processing is sensitive to perceptual depth. The fact that there was no main effect for depth plane showed that this sensitivity can not be interpreted globally. Thus, optimal display location appears to depend on certain characteristics of the informational content, e.g. task type. However, in order to generalize the findings for the detection and the arithmetic task to the task types experiential and reflective, more sample tasks will have to be analysed in experiment two.

\section{EXPERIMENT 2}

The second experiment was concerned with the generalization of task-specificity in peri- and extrapersonal space. The initial Smiley task was replaced by a variation of the d2-test [11], a standard attention and concentration test, where double-primed d's had to be detected among different d- and p-distractors. The arithmetic test was replaced by a standard test on verbal intelligence, where single words have to be evaluated according to their membership to certain groups of words.

Additionally the question whether presenting dual-task information in two different depth planes is at all advantageous to presenting all information in just one depth plane, was examined.

\subsection{Participants}

The test sample consisted of 44 subjects ( 24 female) with a mean age of 29.2 (range: 19-70 years), which were recruited via PESA-subject database, as in experiment one.

\subsection{Procedure and Material}

Subjects were welcomed, briefed and then situated in front of the HoloPro ${ }^{\mathrm{TM}} 3$ i-PrOS Touch within hand-reaching distance. The experimental set-up was equal to the set-up of experiment one.

Before the dual-task trials started, subjects completed five baseline trials of two minutes length each in order to get used to the setting and the tasks (see Figure 3). The first baseline was an object detection task. D's and p's as used in the d2-test were presented randomly every five seconds. Subjects were only to react to double-primed d's by saying "yes" whenever they detected one. Stimuli then disappeared. The second baseline was the same task, but stimuli and distractors were presented at the far screen. The third baseline was a verbal intelligence task. A single word was presented at the near screen (e.g. "hail"), timely followed by a group of words (e.g. "snow, sleet, rain") and subjects were to state via a button-click whether the word presented first could be associated with the group of words. The fourth baseline was the same task, but words were presented at the far screen. The fifth baseline was the arithmetic task from experiment one. Numbers were presented at the near screen. After the baseline phase, four 3 min-long experimental trials followed where the object detection and the arithmetic or verbal tasks had to be completed in parallel. In trial one, d's and p's were presented on the near screen, while words were presented on the far screen. In trial two, screens were switched. In trial three, the smiley-task from experiment one and the arithmetic task were both presented at the near screen, while in trial four, both tasks were presented at the far screen.

When subjects had completed the last trial (approximately after $45 \mathrm{~min})$, they were informed about the purpose of the experiment in more detail, rewarded and dismissed.

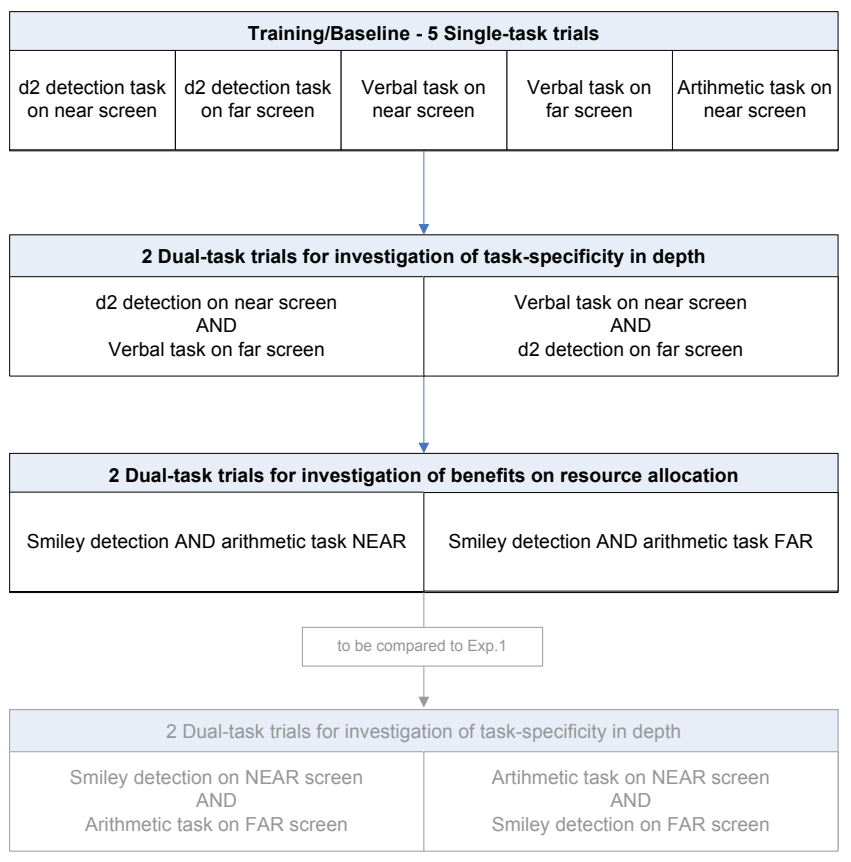

Figure 3. Detailed procedure of experiment two

\subsection{Results}

Data was analysed using SPSS. Group means were compared via analyses of variance (ANOVA) and differences were further investigated using t-tests.

\subsubsection{Task-Specificity}

Means and standard deviations for reaction times in dual-task trials one and two are reported in Table 2. Again, as expected, there was a significant main effect of task $(F(1,43)=227.94$, p $<.001$, partial $\left.\eta^{2}=.841\right)$, meaning that reaction times for the detection task were significantly smaller than those for the verbal task. There was no significant main effect of depth plane and there was no significant interaction effect.

Table 2. Means and standard deviations (SD) for reaction times in seconds, experiment two

\begin{tabular}{|c|c|c|}
\hline & Mean & SD \\
\hline Detection task near & 1.023 & .210 \\
\hline Detection task far & 1.082 & .244 \\
\hline Verbal task near & 1.600 & .334 \\
\hline Verbal task far & 1.630 & .368 \\
\hline
\end{tabular}

\subsubsection{Resource Allocation}

Data relevant for examining resource allocation over two depth planes comes from both experiments (smiley and arithmetic tasks respectively). Means were analyzed via ANOVA also, but only one factor, plane, was examined. Plane has two grades and is defined as follows: (1) Visual information for concurrent tasks is presented in one plane, i.e. either the HoloPro ${ }^{\mathrm{TM}}$ or the 
far screen and (2) information is presented on both screens, as described above.

Figure 4 shows mean reaction times for the single-depth trials (Experiment 2) and the trials where concurrent information was presented on two different displays (Experiment 1).

Statistical analyses did not reveal a significant difference in reaction time for the arithmetic task. This also holds true for the separate analysis of near and far plane trials.

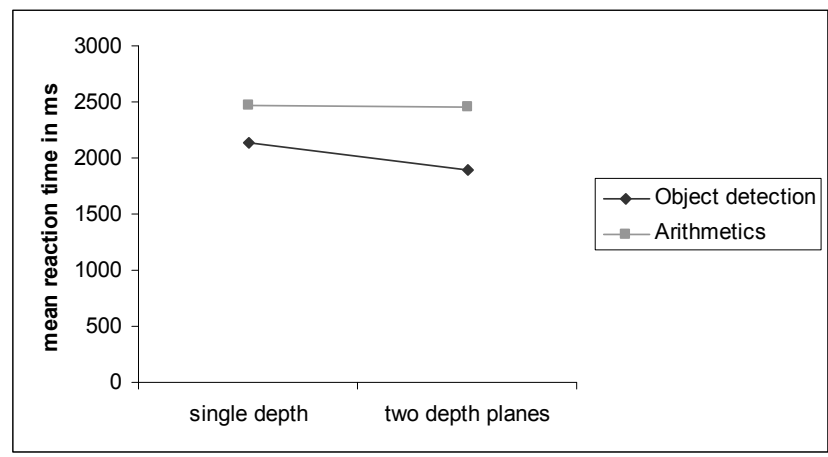

Figure 4. Means for single-depth and two-depths trials

However, there was a significant effect of plane for the object detection task $\left(F(1,87)=5.84, \mathrm{p}<.05\right.$, partial $\left.\eta^{2}=.063\right)$. Reaction times were smaller when two depth planes were utilized for information presentation. This effect is stronger for the near plane conditions $\left(F(1,87)=8.2, \mathrm{p}<.01\right.$, partial $\eta^{2}=$ $.086)$ and only by trend to be seen for the far plane conditions $\left(F(1,87)=3.34, \mathrm{p}<.1\right.$, partial $\left.\eta^{2}=.037\right)$.

\subsection{Discussion}

Results on task-specificity from experiment one could not be generalized in this second experiment. Even though arithmetics and the verbal task can both be considered to be cognitively demanding and part of the reflective cognition category, the verbal task was not sensitive to depth plane. The same holds true for object detection tasks in experiment one and two: They can both be defined as being part of the experiential cognition category but are unequally sensitive to perceptual depth. The reason for this difference might be found in the different level of difficulty of the two tasks. While in experiment one subjects just had to react whenever they noticed something on the screen, in experiment two, they had to first determine whether the appearing object was the target object. D's and p's where paced at every five seconds instead of appearing randomly every one to nine seconds as smileys did in experiment one. This variation had the goal of keeping the difficulty levels about equal, but this attempt obviously failed, which can be observed in different reaction times for the two tasks also.

Concerning resource allocation, results show that the notion of a two-plane benefit is reasonable. However, an effect could only be observed for the object detection task. This problem is worthwhile being investigated further.

\section{GENERAL DISCUSSION}

Results from the two experiments presented in this paper show that human visual information processing is sensitive to perceptual depth. Apparently, this sensitivity is due to the characteristics of a task. However, these characteristics still need to be defined further. A simple classification into experiential and reflective cognition seems to be insufficient. For experiential tasks, difficulty (i.e. cognitive engagement or the closeness to the border between experiential and reflective cognition) seems to play an important role, while for reflective tasks, the type of intelligence utilized to solve the problem appears to have an influence. Task-specificity in processing information in different perceptual depths could be satisfactorily shown for arithmetics, but not for the verbal task. This could be due to the fact that the arithmetic task utilized mathematical intelligence, while the verbal task utilized verbal intelligence. A third experiment, which is currently being designed, includes tasks which utilize figural intelligence, to examine the reflective cognition category further. Additionally, another object detection task will lead to more insights into the experiential cognition category.

\section{ACKNOWLEDGMENTS}

This research is supported by the German Research Foundation (DFG) as part of the Research Training Group 'Prospective Design of Human-Technology Interaction' (no. 1013).

\section{REFERENCES}

[1] He, Z.J. and Nakayama, K. 1995. Visual attention to surfaces in three-dimensional space. Proc. Natl. Acad. Sci. $92,24,11155-11159$.

[2] Arnott, S.R. and Sheffen, J.M. 2000. Attention switching in depth using random-dot autostereograms: Attention gradient asymmetris. Perception \& Psychophysics 62, 7 , 1459-1473.

[3] Keehner, M., Montello, D.R., Hegarty, M. and Cohen, C. 2004. Effects of interactivity and spatial ability on the comprehension of spatial relations in a 3D computer visualization. Presented at the annual meeting of the Cognitive Science Society, Chicago, IL.

[4] Ouerhani, N. and Hügli, H. 2000. Computing visual attention from scene depth, Proc. of $15^{\text {th }}$ Int. Conf. on Pattern Recognition.

[5] Horrey, W.J., Wickens, C.D. and Consalus, K.P. 2006. Modeling Driver's Visual Attention Allocation While Interacting With In-Vehicle Technologies. Journal of Experimental Psychology: Applied 12, 2, 67-78.

[6] Previc, F.H. 1998. The Neuropsychology of 3-D Space Psychological Bulletin 124, 2, 123-164.

[7] McCourt, M.E. and Garlinghouse, M. 2000. Asymmetries of visuospatial attention are modulated by viewing distance and visual field elevation: Pseudoneglect in peripersonal and extrapersonal space. Cortex 36, 5, 715731.

[8] Weiss, P.H., Marshall, J.C., Wunderlich, G., Tellmann, L., Halligan, P.W., Freund, H.-J., Zilles, K., and Fink, G.R. 2000. Neural consequences of acting in near versus far space: a physiological basis for clinical dissociation. Brain, 123, 2531-2541.

[9] Norman, D.A. 1993. Things that Make Us Smart. Addison-Wesley Publishing Company.

[10] Wickens, C.D. 2002. Multiple resources and performance prediction. Theoretical Issues in Ergonomics Science 3, 2, 159-177.

[11] Brickenkamp, R. 2002. Handbuch psychologischer und pädagogischer Tests (3., vollst. überarb. und erw. Aufl.). Hogrefe. 